\title{
Belphégor
}

Littérature populaire et culture médiatique

10-3 | 2011

Peter Pan

\section{Le mythe de Peter Pan ou l'angoisse du temps qui passe}

Amélie Maxwell

\section{OpenEdition}

Journals

Édition électronique

URL : http://journals.openedition.org/belphegor/389

DOI : 10.4000/belphegor.389

ISSN : 1499-7185

Éditeur

LPCM

Édition imprimée

Date de publication : 10 janvier 2011

\section{Référence électronique}

Amélie Maxwell, « Le mythe de Peter Pan ou l'angoisse du temps qui passe », Belphégor [En ligne],

10-3 | 2011, mis en ligne le 10 janvier 2013, consulté le 19 avril 2019. URL : http://

journals.openedition.org/belphegor/389 ; DOI : 10.4000/belphegor.389

Ce document a été généré automatiquement le 19 avril 2019.

\section{(c) (i) $(9$}

Belphégor est mis à disposition selon les termes de la Licence Creative Commons Attribution - Pas d'Utilisation Commerciale - Pas de Modification 4.0 International. 


\title{
Le mythe de Peter Pan ou l'angoisse du temps qui passe
}

\author{
Amélie Maxwell
}

« [L]e sentiment d'aventure ne vient décidément pas des événements : la preuve en est faite. C'est plutôt la façon dont les instants s'enchaînent. Voilà, je pense, ce qui se passe : brusquement on sent que le temps s'écoule, que chaque instant conduit à un autre instant, celui-ci à un autre et ainsi de suite ; que chaque instant s'anéantit, que ce n'est pas la peine d'essayer de le retenir, etc. Et alors on attribue cette propriété aux événements

qui vous apparaissent dans les instants ; ce qui appartient à la forme, on le reporte sur le contenu. En somme, ce fameux écoulement du temps, on en parle beaucoup, mais on ne le voit guère. On voit une femme, on pense qu'elle sera vieille, seulement on ne la voit pas vieillir. Mais, par moments, il semble qu'on la voie vieillir et qu'on se sente vieillir avec elle : c'est le sentiment d'aventure. On appelle ça, si je me souviens bien,

l'irréversibilité du temps. Le sentiment de

l'aventure serait, tout simplement, celui de l'irréversibilité du temps. Mais pourquoi est-ce qu'on ne l'a pas toujours? Est-ce que le temps ne serait pas toujours irréversible? Il y a des moments où on a l'impression qu'on peut faire ce qu'on veut, aller de l'avant ou revenir en arrière, que ça n'a pas d'importance; et puis d'autres où

l'on dirait que les mailles se sont resserrées et, 
dans ces cas-là, il ne s'agit pas de manquer son coup parce qu'on ne pourrait plus recommencer. » Jean-Paul Sartre, La nausée

1 Les migrations de personnages célèbres de la page à l'écran attestent une chose : bien qu'ils y soient souvent nés, «les mythes se situent hors du texte ${ }^{1}$ »; ils n'en sont pas prisonniers. En effet, les mythes traversent les frontières, dont l'évidente frontière du temps, pour en arriver à exister dans l'imaginaire collectif indépendamment de leur créateur ou de leur œuvre d'origine, qu'ils dépassent alors en popularité. Or, comment un personnage donné devient-il une référence culturelle si répandue? Qu'est-ce qui fait en sorte que ce personnage soit promu au rang de mythe alors que tant d'autres sont oubliés, voire passent inaperçus ? Pouvons-nous lui attribuer tout le mérite ou celui-ci revient-il à l'histoire dans laquelle il apparaît, à son auteur fondateur, à ses adaptations, à son époque de parution, à son public? Manifestement, «[1]'emprise de tels personnages sur l'imaginaire collectif soulève plusieurs questions tant sur leur contexte d'origine que sur les conditions de leur longévité virtuelle ${ }^{2} »$. Et ces questions, en plus d'expliquer la pérennité de ces personnages devenus mythes, dévoilent les plus profondes préoccupations de l'humanité, avec artifice, oui, mais sans censure.

2 Ainsi, quoiqu'il soit souvent issu de la fiction, « [1]e mythe est une histoire vraie, c'est-àdire plus vraie que ne l'est la réalité d'ici-bas, qui raconte, explique et justifie l'existence du monde et l'existence de l'homme dans leurs déterminations essentielles ${ }^{3} »$. En d'autres mots, pour avoir un succès à ce point retentissant, quelque chose dans cette histoire parle des lecteurs aux lecteurs, révèle les phénomènes sur lesquels s'interrogent les hommes de façon récurrente. En tentant donc d'entrer en communication avec les lecteurs/ spectateurs, une telle histoire ne peut guère se limiter à un auditoire d'élite. Au contraire, les questions que pose le mythe s'adressent à tous les hommes qui s'y intéressent, qui cherchent à participer au dialogue qu'il tente d'établir. Le mythe a donc une grande utilité pour "[1]'homme [qui] veut comprendre l'univers, [qui] veut le comprendre comme un tout mais aussi dans ses phénomènes [...]. Quand l'univers se crée ainsi à l'homme par question et par réponse, une forme prend place, que nous appellerons mythe $e^{4}$ ». C'est donc dire que l'homme qui s'intéresse au mythe s'intéresse à lui-même et à ses semblables, puisque mythe et humanité sont indissociables.

3 Et comme question et réponse sont aussi indissociables, ou du moins nous aimerions qu'il en soit ainsi, l'homme «tient ce qu'il ne comprend pas pour une ancienne réponse, à laquelle il reste simplement à chercher la question qui lui convienne. S'il en est bien ainsi, on comprend que dans un tel contexte les questions soient plus significatives que les prétendues réponses ${ }^{5} »$. Cela ne veut pas pour autant dire que les réponses, variant d'une époque à l'autre selon les valeurs en vogue, ne sont d'aucun intérêt, mais seulement qu'elles ne suffisent pas à transformer une histoire ou un personnage en mythe. Davantage que la clé du mystère, c'est le mystère lui-même qui produit un effet sur nous, qui constitue le cœur du mythe.

Bref, ce sont les questions qu'il pose, l'appel qu'il nous lance qui créent l'attrait d'une histoire ou d'un personnage. Prenons par exemple le mythe de Peter Pan. Ce texte a interpellé les masses dès son apparition et continue de les agripper aujourd'hui, plus de cent ans plus tard. Depuis la plume de James Barrie, Peter Pan a muté de la page au grand écran dans plusieurs versions subséquentes du mythe. De celles-ci, l'adaptation du mythe de Walt Disney et la réinterprétation du mythe de Steven Spielberg sont sans doute les plus connues, contribuant ainsi à la survie de Peter Pan. C'est donc avec une perspective 
mythocritique que nous aborderons le roman de 1911 de Barrie ${ }^{6}$, pierre angulaire de cette analyse. À cette œuvre, nous comparerons le dessin animé de 1953 de Disney $^{7}$ et le film de 1991 de Spielberg ${ }^{8}$, qui par leurs différences en dévoilent beaucoup sur nous, les spectateurs. Pour ce faire, nous prendrons comme porte d'entrée les questions qui constituent l'essence du mythe de Peter Pan, questions qui sont à la fois les raisons de sa survie et de son succès d'origine, questions qui cachent certaines de nos préoccupations fondamentales liées au temps, à son caractère insaisissable et à notre capacité ou non d'y échapper.

\section{Peter Pan raconte ${ }^{9}$}

$5 \quad$ Hantés par ces mystérieuses préoccupations, nous tentons tant bien que mal d'en dégager les solutions. Et pour preuve, du mystère, une force tout aussi attirante qu'insupportable, découle des solutions non seulement sous forme de religion et de science, mais aussi sous forme d'art, puisque «c'est précisément du mystère que [naît] le mythe ${ }^{10}$ ». Nous pouvons donc dire que Peter Pan sert d'interprète à un de nos plus profonds questionnements : sommes-nous à la merci du temps ? Notons par ailleurs que, dans la littérature, «[o]n devine [...] une révolte contre le temps historique, le désir d'accéder à d'autres rythmes temporels que celui dans lequel on est obligé de vivre et de travailler. [...] Tant que subsiste ce désir, on peut dire que l'homme moderne garde [...] certains résidus d'un "comportement mythique"11.» Il est donc clair que le temps est ce mystère à la fois au cœur du mythe et de sa pérennité.

Depuis sa naissance il y a plus d'un siècle, Peter Pan est resté jeune dans nos esprits, à une exception près : Hook, film dans lequel Spielberg projette Peter Pan dans le monde adulte. En effet, alors que le Peter Pan de Disney, certes édulcoré, se veut une adaptation cinématographique du texte de Barrie, le Peter Pan de Spielberg, devenu un homme, remet en question le texte de Barrie, dans lequel il ne grandit jamais. Quoiqu'intéressant, le Peter Pan adulte de Spielberg n'a pas supplanté le Peter Pan enfant que Barrie - aidé de Disney - nous a légué. Ainsi, tel que nous voudrions l'être, le personnage de Peter Pan et le mythe de Peter Pan sont tous les deux à l'épreuve du temps.

\section{Réalité désagréable}

7 Avant que l'histoire de Peter Pan telle que nous la connaissons ne commence, ce personnage n'avait rien d'extraordinaire, c'est-à-dire qu'il était un enfant comme les autres, comme nous autres. Cependant, la suite de son enfance n'est pas la même que la nôtre: elle ne débouche jamais sur l'adolescence puis l'âge adulte. C'est ainsi que, déterminé à larguer un monde qui l'assommerait certainement du poids des responsabilités augmentant invariablement avec le temps, il largue les amarres dans un monde où il est « the actor-director ${ }^{12}$ », donc où il arrive à déjouer les lois du temps pour en faire son havre intemporel.

Or, que ce soit au Pays Imaginaire, lieu qui obéit à sa volonté de freinage, ou à Londres, lieu où il se plaît à écouter les histoires que Wendy raconte à ses frères, l'enfant éternel porte bien ce sobriquet. Bref, peu importe le monde où il se trouve et les lois qui le régissent, Peter Pan ressent le besoin urgent d'abandonner la réalité pour s'abandonner à une fantaisie où il arrive à s'extraire du temps historique. Ce n'est toutefois pas le cas des enfants Darling, que le temps menace d'enlever à leurs jeux. Voilà sans doute pourquoi la 
concrétisation du concept généralement abstrait du temps ne s'opère que lorsque Wendy se joint à Peter Pan, moment qui coïncide avec celui où nous, lecteur/spectateurs, nous joignons également à Peter Pan. En effet, nous pouvons dire que l'envolée des enfants Darling guidée par Peter Pan sert d'assise au mythe, car c'est leur départ tout autant que le nôtre de Londres - une ville gouvernée par le temps comme en témoigne Big Ben au sommet du parlement britannique - qui illustre notre désir d'évasion, de maîtrise du temps.

Le recul que prennent les enfants Darling du vrai monde, et donc du temps, est alors reflété chez les lecteurs/spectateurs qui, lorsqu'ils s'immergent dans l'œuvre, peuvent eux aussi prendre du recul vis-à-vis du vrai monde, et donc du temps, pour l'aborder avec plus de lucidité1 ${ }^{13}$. Or, nous ne devons pas cette scène profondément marquante à l'auteur fondateur du mythe de Peter Pan, mais bien à son vecteur mémoriel ${ }^{14}$ : le dessin animé de Disney. En effet, bien que Barrie ait trouvé le slogan du mythe, n'oublions pas que nous devons son emblème, sa notoriété, à Disney ${ }^{15}$. C'est donc cette version du mythe dans laquelle les enfants se posent sur les aiguilles de Big Ben qui sert d'ancrage au mythe de Peter Pan dans l'imaginaire collectif, puisque c'est grâce à cette image devenue marque de commerce que le mythe communique avec l'humanité.

Par ailleurs, contrairement à un roman, un dessin animé a la possibilité de superposer plusieurs éléments marquants afin d'intensifier le rapport du spectateur avec la scène dont il est question. Comme pour les nombreuses autres histoires desquelles il a fait un mythe, Disney s'empare encore une fois de cette occasion pour prouver que les films, peut-être davantage que les livres, «ont quelque chose de magique ${ }^{16}$ ». Notamment, à l'image regroupant Peter Pan, les enfants Darling et Big Ben, est ajoutée la chansonthème du mythe, dont les paroles ne sont pas sans importance puisqu'elles témoignent de la légèreté de l'enfance exempte de responsabilités que Peter Pan cherche à faire durer toujours : " Think of all the joy you'll find, When you leave the world behind and bid your cares goodbye, You can fly!» (Disney) Ainsi, nous obtenons le montage suivant: une chanson encourageant les spectateurs à se libérer de leurs responsabilités d'adulte, une image montrant des enfants qui s'envolent vers un autre monde après avoir détraqué le temps du nôtre, et un slogan indiquant la renaissance de ceux qui explorent cet autre monde : "Second star to the right, and straight on till morning. " (Barrie, p. 50) Or, étant donné que «[c]artoons provide opportunities to escape the constraints of the material world, and [...] also evoke childhood pleasures ${ }^{17}$ ", cette qualité du cinéma est d'autant plus importante dans la mythisation de Peter Pan.

\section{Fiction agréable}

11 Comme nous le savons tous, ce n'est pour nulle autre raison que de pouvoir dicter luimême les lois du temps et diriger « a frightful sneer at the laws of nature » (Barrie, p. 212) que Peter Pan quitte Londres et son infiniment célèbre Big Ben pour le Pays Imaginaire, lieu où il est possible de réaliser l'égoïste rêve qui définit son personnage, où il se rassure d'être : « exactly the same as ever » (Barrie, p. 228) malgré le temps qui passe. Ainsi, ce que nous retenons invariablement de Peter Pan, c'est la moquerie qu'il fait du temps historique en esquivant complètement son effet sur les hommes : l'âge adulte.

12 Toutefois, cela n'est pas le cas dans la version plus distanciée que propose Spielberg. Même le texte de Barrie suggère que Peter Pan n'est pas complètement à l'abri des violences du temps : « Sometimes, though not often, he had dreams, and they were more 
painful than the dreams of other boys. For hours he could not be separated from these dreams, though he wailed so piteously in them. They had to do, I think, with the riddle of his existence.» (Barrie, p.166-167) Par ailleurs, il ne faut pas oublier que Peter Pan partage le refuge qu'il a lui-même créé avec le capitaine Crochet, un terrible pirate qui le pourchasse et qui est en retour pourchassé par un crocodile affamé agissant comme un réveille-matin ambulant. C'est donc dire que, «[1]ike the author of children's literature, Peter kidnaps the child to escape the nursery, gravity and other limitations and burdens of ordinary reality; but he takes us to a world infused with morbid reminders of that reality ${ }^{18}$ ». Ainsi, malgré le fait que le Pays Imaginaire exerce un pouvoir revitalisant sur Peter Pan, il n'est pas pour autant imperméable aux différents rappels du temps que l'enfant refuse, fuit, mais qui s'immiscent tout de même dans un monde créé pour les éviter. La peur que nous inspire le temps est donc omniprésente dans ce mythe, et ce surtout dans Hook, où « Spielberg fills the mise-en-scène with an almost numbing variety of time pieces, from postcard-perfect shots of Big Ben [...] to Hook's (Dustin Hoffman) emblematic museum of broken clocks ${ }^{19}$ ». L'incompatibilité paradoxale entre temps et enfance apparaît donc dans plusieurs aspects du mythe : au sein du personnage même de Peter Pan, dans l'écart entre le Pays Imaginaire et Londres, puis à travers le combat entre Peter Pan et le capitaine Crochet.

En effet, même si d'importantes caractéristiques communes unissent ces deux personnages, Barrie les oppose en traçant un « war-path » (Barrie, p. 70) entre eux. L'un représentant l'enfant, l'autre, la menace des ravages du temps sur le corps et l'esprit, Barrie demande clairement aux lecteurs : «Which will win?» (Barrie, p. 70) Ce combat central à toutes les versions du mythe nous met à l'affut à la fois du dialogue qu'il tente d'établir avec l'humanité et de la contradiction au cœur du personnage de Peter Pan. En effet, sa révolte contre le temps est si puissante qu'elle fait en sorte qu'il quitte Londres pour un monde qui outrepasse la frontière du temps alors qu'il en traîne la trace avec lui ; une trace qui le suit comme un crochet profondément ancré dans sa proie : la présence du capitaine Crochet au Pays Imaginaire. Craignant donc la même chose - la mort - mais ne s'en doutant probablement pas, ils se battent plutôt que de s'épauler: "'Proud and insolent youth', said Hook, 'prepare to meet thy doom.' 'Dark and sinister man,' Peter answered, 'have at thee.' » (Barrie, p. 197) Ils agissent sans doute de façon antagoniste pour se réconforter, pour infliger à l'autre ce à quoi ils ont tous les deux peur de succomber et ainsi s'en montrer plus fort : la mort.

En plus de cet affront entre le pirate et l'enfant, la porosité du Pays Imaginaire témoigne donc des variations idéologiques d'une époque à l'autre depuis la parution de Peter Pan. En effet, une ligne de temps illustrant les fluctuations de la valeur réservée aux enfants et aux adultes se forme depuis le roman de Barrie du début du $\mathrm{xx}^{\mathrm{e}}$ siècle (où nous sentons une véritable tension chez le narrateur rêveur et sceptique à la fois), jusqu'au dessin animé de Disney (où le voile dubitatif de la version originale est levé et la promotion de l'enfance bat son plein), jusqu'au film de Spielberg de la fin du $\mathrm{xx}^{\mathrm{e}}$ siècle (qui, tout en célébrant les joies de l'enfance, n'ignore pas la valeur des responsabilités de l'âge adulte). Dans ce film, le capitaine Crochet tente de sensibiliser Peter Pan à l'importance de son rôle de vilain en lui demandant: "What would the world become without Captain Hook?» (Spielberg) Il est donc d'autant plus clair que ce mythe ne raconte pas que l'histoire de Peter Pan, mais la nôtre également. 


\section{Peter Pan révèle}

15 Avant de poursuivre, rappelons qu'un mythe ne se résume pas qu'à l'acte de raconter une histoire ; la clé n'est pas là. Plutôt, « [s]a force vient de son aptitude à interpeller [...] les spectateurs ou les lecteurs, et à trouver parmi eux, éventuellement - car une telle parole n'est pas toujours entendue, ni même, à certaines époques, audible -, celui qui s'emparera d'elle et continuera à la faire vivre ${ }^{20} »$. Ayant trouvé ces voix pour perpétuer le mystère qui l'anime, Peter Pan révèle le «thème mythique ${ }^{21}$ " qui fait sa force; le thème qui, comme en témoigne sa récurrence chez tous les porte-parole du mythe, continue de fasciner par son caractère insaisissable : le temps. C'est donc grâce à un enfant, à un pirate et à un crocodile que Barrie a initié au début $\mathrm{du} \mathrm{xx}^{\mathrm{e}}$ siècle un dialogue entre mythe et humanité.

Or, si nous tenons pour vrai que « $[\mathrm{m}]$ ythology has a great deal to do with the stages of life, the initiation ceremonies as you move from childhood to adult responsibilities ${ }^{22}$ ", il n'est pas surprenant que des voix aussi puissantes que celles de Disney et de Spielberg aient relancé le dialogue abordé par Barrie, faisant en sorte que Peter Pan échappe aux prises de son créateur et se diffuse dans la littérature, le cinéma et l'esprit des lecteurs/ spectateurs. Ainsi propulsé dans l'imaginaire collectif, Peter Pan conteste l'inquiétant pouvoir du temps sur les hommes puisqu'il ne subit pas les transformations que celui-ci inflige à ses lecteurs/spectateurs. Opposant donc pérennité et finitude, Barrie, Disney et Spielberg cherchent les réponses aux questions suivantes.

\section{Qu'est-ce que le temps?}

Davantage que le temps historique qui concerne l'humanité, c'est le temps personnel qui ne regarde que lui-même qui inquiète Peter Pan - et qui nous inquiète également. Or, la dimension profondément humaine du mythe qui incorpore nos préoccupations réelles à une histoire de fiction montre à quel point un questionnement est puissant lorsqu'il concerne tous les hommes individuellement. En effet, alors que la question est posée par l'humanité, chaque homme y cherche une réponse qui lui convienne personnellement, ce qui fait que tous les lecteurs/spectateurs se sentent intimement liés à l'appel collectif du mythe. Cet effet égocentrique du mythe exerce une grande force d'attraction, comme en témoigne la popularité de Peter Pan. En effet, un des atouts du mythe de Peter Pan est qu'il invite les lecteurs/spectateurs à réfléchir sur l'essence du temps d'un point de vue strictement humain, centrant donc le questionnement des lecteurs/spectateurs sur euxmêmes. Qui plus est, le mythe de Peter Pan non seulement aborde le temps grâce à sa manifestation humaine (le vieillissement), mais incite les lecteurs/spectateurs à se placer eux-mêmes sur le "continuum du temps humain ${ }^{23}$ ", suggérant donc l'inhabituelle possibilité d'y avancer et d'y reculer. En effet, Barrie et Disney placent Peter Pan d'un côté et le capitaine Crochet de l'autre, permettant uniquement à Wendy d'osciller entre les deux pôles en ne laissant qu'à elle seule le pouvoir de s'y mouvoir grâce à la mémoire. C'est plus complexe chez Spielberg. Tel que nous le verrons plus tard, celui-ci n'oppose pas les personnages d'une façon aussi antagoniste, ni selon une vision aussi manichéenne. Par ailleurs, les trois versions du mythe cherchent à hiérarchiser l'enfance par rapport à l'âge adulte, étant toutes confrontées à la question de l'œuf ou la poule. Bien que nous croyions que la jeunesse et la vieillesse occupent chacune de leur propre extrémité du 
continuum du temps humain, nous ignorons quel état découle duquel. Si un enfant se transforme en adulte au fil des ans et un adulte engendre un enfant au fil des mois, lequel est apparu en premier: l'enfant ou l'adulte? Peter Pan, tout vêtu de vert comme d'ignorance, ou le capitaine Crochet, terrible pirate traqué par un réveille-matin reptilien? Lequel de ces deux personnages, toujours rencontrés sous forme de duo, a-t-il été créé en réponse à l'autre?

Du reste, qu'entendons-nous par enfant et adulte ? Pour le bien de notre réflexion, faisons la simple mais importante distinction suivante : l'adulte est généralement vu comme une personne très consciente, voire obsédée par le temps qui défile, alors que l'enfant ne s'en préoccupe généralement pas, croyant avoir une éternité de jeux devant lui. Par contre, si le temps n'a aucune importance aux yeux des enfants du seul fait qu'ils ignorent ce que c'est, Peter Pan est un cas à part. Sans parler de ses caractéristiques fantastiques, ce qui est particulier chez Peter Pan est ceci : bien qu'il n'ait « no sense of time» (Barrie, p. 221), il est inquiet devant la possibilité qu'il doive un jour franchir une mystérieuse frontière l'ayant jusque là maintenu en enfance. En effet, il ne connaît pas son âge, mais il s'accroche de tout son être à l'enfance et trouve insupportable l'idée de devenir un homme, cette créature à connotation négative qui lui rappelle la précarité de l'innocence. En cela, Peter Pan et le capitaine Crochet sont semblables, puisque ce dernier est également préoccupé par la puissance du temps, fuyant le son du crocodile qui le traque en faisant tic tac. Ce son répétitif et récurrent est tout sauf banal : produisant une très grande agitation chez le capitaine Crochet, ce tic tac lui rappelle constamment que les aiguilles du temps s'agitent en son prédateur... ainsi qu'en lui. Or, non seulement le crocodile ne pourchasse que le capitaine Crochet - ciblant le pirate comme s'il sentait sa peur -, mais il continue plus avidement encore de le pourchasser après y avoir goûté, après avoir mangé la main droite que Peter Pan lui a coupée : «It liked my arm so much, Smee, that it has followed me ever since, from sea to sea and from land to land, licking its lips at the rest of me. » (Barrie, p. 76) Ainsi, la présence du crocodile concrétise les effets incontournables, donc angoissants, du temps, qui a déjà pris une bouchée du capitaine Crochet, une bouchée qui définit ce personnage ${ }^{24}$ dans l'imaginaire collectif.

Il est donc intéressant d'observer qu'au coeur des deux personnages que nous essayons d'opposer sur le continuum du temps humain est ancrée la même préoccupation: "[...] each fears the passage of time with the inevitable changes and transformations it occasions ${ }^{25}$ ", cherchant par conséquent à éviter ses ravages. Cependant, derrière cette même peur se cache deux angoisses différentes. Alors que Peter Pan craint la mort de l' esprit avec l'âge, le capitaine Crochet craint la mort du corps.

\section{Pouvons-nous échapper aux prises du temps?}

21 Encore une fois différent des autres enfants, Peter Pan est orphelin par choix, s'étant exilé loin de toute personne pouvant l'élever en homme. En effet, si Peter Pan refuse de grandir, c'est parce qu'il perçoit le temps comme une menace aux plaisirs de l'enfance, à la légèreté de l'esprit. Ne craignant donc pas tant la mort du corps - ou ne la concevant peut-être simplement pas à cause de son arrêt de croissance -, Peter Pan multiplie les combats contre le capitaine Crochet, «a cadaverous man » (Barrie, p. 69) éveillant en lui la possibilité de la mort de l'esprit. Or, notons que Peter Pan repousse les menaces à ses plaisirs d'enfance avec beaucoup plus de violence dans le roman de Barrie que dans les films ultérieurs. Cette violence est sans doute due au fait que le souhait de Peter Pan est 
plus difficile à réaliser dans ce roman du début $d u x^{e}$ siècle, où il est le seul enfant à rester jeune au Pays Imaginaire, comme si l'âge adulte était une contagion. Le Peter Pan de Barrie ne s'attaque donc pas qu'aux pirates, mais aussi à la menace qu'est devenue sa propre bande : «[W]hen they seem to be growing up, which is against the rules, Peter thins them out. » (Barrie, p. 66) Qui plus est, il n'y a pas que le capitaine Crochet ou les garçons perdus de Barrie qui tentent de modérer les élans épicuriens de Peter Pan, mais aussi Mrs. Darling :

She told Peter that she had adopted all the other boys, and would like to adopt him also. 'Would you send me to school ?' he enquired craftily. 'Yes.' 'And then to an office?' 'I suppose so.' ‘Soon I should be a man?' 'Very soon.' 'I don't want to go to school and learn solemn things,' he told her passionately. 'I don't want to be a man. Oh, Wendy's mother, if I was to wake up and feel there was a beard!' [...] Mrs Darling stretched out her arms to him, but he repulsed her. 'Keep back, lady, no one is going to catch me and make me a man.' (Barrie, p. 219)

Après s'être enfui de sa propre mère, Peter Pan continue donc de rejeter toute figure parentale à l'exception de Wendy, qu'il ramène avec lui au Pays Imaginaire afin qu'elle serve de mère aux garçons perdus.

Toutefois, Wendy ne joue pas véritablement le rôle d'une mère ; elle raconte des histoires aux garçons au lieu de les initier à l'âge adulte par l'éducation, prolongeant ainsi leur enfance. En effet, étant elle-même une enfant, Wendy maintient les garçons perdus dans un monde de fantaisie plutôt que de les élever comme le ferait Mrs. Darling, c'est-à-dire selon les codes de conduite contraignants prescrits par la société du début du $\mathrm{xx}^{\mathrm{e}}$ siècle qui valorisent le passage de l'enfance à l'âge adulte :

Mrs. Darling first heard of Peter when she was tidying up her children's minds. It is the nightly custom of every good mother after her children are asleep to rummage in their minds and put things straight for the next morning, repacking into their proper places the many articles that have wandered during the day. (Barrie, p. 6-7)

Sans mère pour le guider, Peter Pan contourne donc les responsabilités alourdissant l'enfant jusqu'à l'âge adulte, et réussit à vivre une perpétuelle enfance. Cette enfance est cependant interrompue chez Spielberg, qui choisit de défier à la fois la version originale $\mathrm{du}$ mythe et son vecteur mémoriel en mettant en scène un Peter Pan adulte plutôt qu'enfant. Le contraste évident entre Peter Banning (l'adulte sérieux) et Peter Pan (l'adulte espiègle) représente parfaitement la hantise de Peter Pan : non pas vieillir, mais grandir ${ }^{26}$. Notons toutefois que le Peter Pan de Spielberg est joué par Robin Williams, ce qui n'est pas peu de choses. Sans doute choisi pour sa verve, parce qu'il s'éloigne de l'idée que nous nous faisons d'un adulte sérieux et responsable, cet acteur incarne l'idéal du rôle. En effet, l'enfance perdue puis retrouvée de ce Peter Pan adulte, quoiqu'elle ne soit plus entière, est tout aussi vraie: tel qu'il le souhaitait, il redevient jeune d'esprit et restera ainsi.

Avec le temps, nous observons donc que l'inversion de valeur accordée à l'enfance à et l'âge adulte est reflétée dans les arts. Du Peter Pan acharné et mesquin de Barrie, nous passons au Peter Pan gai et sans souci de Disney, caractéristiques connues du grand public et issues d'une période où l'éloge de l'aspect terre-à-terre de l'âge adulte faisait place à l'éloge de l'aspect aérien de l'enfance. Puis, à la fin $\mathrm{du} \mathrm{xx} \mathrm{x}^{\mathrm{e}}$ siècle, Spielberg met en scène un Peter Pan devenu adulte malgré sa résistance d'autrefois, un adulte représentant exactement ce qu'il craignait devenir mais qui finit par retrouver les joies de l'enfance, suggérant peut-être que le temps soit une force moins périlleuse qu'elle lui paraissait plus jeune. 

positif et que le capitaine Crochet soit le méchant. Le mythe de Peter Pan tente en effet de différencier le fait de grandir et de vieillir, suggérant que nous puissions dans une certaine mesure échapper aux transformations que le temps impose à l'esprit, contrairement à celles qu'il inflige au corps. Et pour preuve, Wendy, même vieille, aime revivre ses aventures d'enfance et continue de croire aux aventures de Peter Pan en attendant son retour, tandis que, malgré ses tentatives de fuites, le capitaine Crochet trouve la mort dans la gueule du crocodile qui était « in dogged pursuit of him » (Barrie, p. 121). Ainsi, aux questions liées à nos préoccupations temporelles, Peter Pan offre les réponses suivantes.

\section{Le pouvoir incontrôlable du temps}

Contrairement à ce que nous pourrions penser de prime abord, le mythe de Peter Pan confirme que le pouvoir du temps sur nos vies est incontrôlable : prisonniers du temps, les enfants Darling vieillissent et le capitaine Crochet meurt. Cependant, il est important 
de savoir que le dessin animé de Disney tait cette réalité inévitable en ne montrant que le retour des enfants Darling à Londres, mais pas leur vie à Londres, de même que la poursuite du capitaine Crochet par le crocodile muni des aiguilles du temps, mais pas le dénouement de leur course. En effet, aucun personnage ne meurt dans cette adaptation du mythe :

[...]this film refutes the idea that childlike imagination might be affected by time or death. Even the crocodile that tracks Captain Hook is not particularly sinister; the film's animation and soundtrack exploit its comic qualities, and it defeats Hook not by eating him but by pursuing him off-screen in a gleefully cartoonish chase ${ }^{29}$.

Peut-être est-ce la raison de la popularité de cette version: plutôt que de donner des réponses claires et précises, le vecteur mémoriel favorise le mystère (donc les questions), gardant les spectateurs en haleine plutôt que de satisfaire pleinement leur curiosité comme le font Barrie et Spielberg. En effet, leur capitaine Crochet est englouti par le crocodile, joignant alors le réveille-matin qui l'y attendait, et leur Wendy devient adulte, ensuite mère, puis grand-mère :

[s] he turned up the light, and Peter saw. He gave a cry of pain and [...] drew back sharply. [...] 'I am old, Peter. I am ever so much more than twenty. I grew up long ago.' 'You promised not to!' 'I couldn't help it.' (Barrie, p. 230)

Ainsi, même les personnages d'une fiction fantastique refoulant la vitesse du temps peuvent en porter les marques, validant de ce fait le fondement de notre préoccupation.

Or, le ton que le narrateur de Barrie réserve aux enfants qui sont devenus des adultes est intéressant et révélateur. D'une part, il semble que clémence et compréhension soient accordées à Wendy, qui a vieilli. D'autre part, mépris et rudesse semblent être le lot des garçons perdus qui ont grandi :

Before they had attended school a week they saw what goats they had been not to remain on the island; but it was too late now, and soon they settled down to being as ordinary as you or me or Jenkins minor. It is sad to have to say that the power to fly gradually left them. [...] In time they could not even fly after their hats. Want of practice, they called it; but what it really meant was that they no longer believed. (Barrie, p. 221-222)

Barrie va même jusqu'à tuer la fée Clochette : symbole des rêves d'enfance qui est la seule à pouvoir, avec Disney, guider le capitaine Crochet vers Peter Pan et, avec Spielberg, guider Peter Banning vers Peter Pan. Qui plus est, bien que Barrie réalise le souhait téméraire de Peter Pan et que celui-ci continue de visiter les descendantes de Wendy, il termine son roman par un avertissement : « [...] and thus it will go on, so long as children are gay and innocent and heartless » (Barrie, p. 232). Barrie est le seul à prendre ce ton envers les lecteurs. Beaucoup plus optimiste, le dessin animé de Disney ne met pas les spectateurs en garde contre l'âge adulte, mais les convainc de la valeur de l'enfance. Beaucoup plus réaliste, le film de Spielberg tâche d'ouvrir de nouveaux horizons.

Par ailleurs, le film de Spielberg accorde plus de temps à la réalité, ce qui nous permet d'aborder l'usure du temps dans les conditions auxquelles nous, les lecteurs/spectateurs, avons droit, nous qui n'avons pas la possibilité de littéralement nous réfugier au Pays Imaginaire. Dans ce film, même Peter Pan est victime de la violence du temps : il grandit, il grossit, il ride, bref son corps de garçon se transforme en corps d'adulte malgré sa résolution d'enfance ; ses nouvelles aventures, c'est-à-dire se marier, avoir des enfants et gagner sa vie, ne sont plus un jeu de make-believe. Il ne peut pas simplement abandonner la partie et en commencer une autre : il a des responsabilités à prendre. Ayant cette fois quitté Londres non pas pour le Pays Imaginaire mais pour les États-Unis, Peter Pan 
s'immerge dans un monde de travail compétitif, vieillit et, incarnant sa peur d'enfance, grandit. Malgré ce "handicap", Peter Banning ${ }^{30}$ devra retourner de force à un Pays Imaginaire qu'il a oublié et auquel il refuse de croire. En sera-t-il de nouveau transformé ?

\section{Le pouvoir revitalisant de l'imaginaire}

37 Dans le roman de Barrie, le Pays Imaginaire somnole en l'absence de Peter Pan et ne reprend vie qu'à son retour : "with the coming of Peter, [...] if you put your ear to the ground [...], you would hear the whole island seething with life» (Barrie, p. 65). Dans le film de Spielberg, c'est l'inverse. Peter Pan est celui qui somnole en l'absence du Pays Imaginaire, et ce à un tel point qu'il devient Peter Banning. En effet, ce n'est qu'au moment où Peter Banning revisite le lieu proliférateur des fantaisies de son enfance prolongée qu'il retrouve le garçon en lui qu'il avait perdu. Ainsi, Spielberg conteste l'incompatibilité entre l'enfance et l'âge adulte que Barrie suggérait. En effet, dans ce roman, Peter Pan ne vit que d'imaginaire et reste grâce à cela jeune à jamais : "Makebelieve was so real to him that during a meal of it you could see him getting rounder.» (Barrie, p. 98-99) Or, en mettant en scène un Peter Pan adulte, Spielberg remet en question l'étanchéité qui séparait les enfants et les adultes en deux mondes chez Barrie en faisant au contraire un parallèle entre eux, puisque Peter Banning redevient Peter Pan même s'il a depuis longtemps dépassé l'adolescence. Qui plus est, cet adulte, ce père de famille, ne retrouve Peter Pan que lorsqu'il retrouve un enfant : son enfant, qui lui donne la bouffée de légèreté dont il avait besoin pour se remettre à voler.

Par ailleurs, Tootles, un garçon perdu qui a quitté le Pays Imaginaire pour revenir à Londres, retrouve la légèreté de l'enfance lorsqu'il retrouve le jeu qu'il cherchait depuis longtemps. Appesanti, vieux et grognon sans ses billes, il ne cesse de répéter, confus : «I lost my marbles.» (Spielberg) Dans ce cas-ci, il est évident que cette expression perd sa signification habituelle (perdre la raison) et renvoie plutôt au vieillissement. En effet, le mot «billes» est dans ce contexte pris au sens propre, évoquant les jeux de l'enfance auxquelles se rattachent tant de "happy thoughts » (Spielberg), qui ont une importance centrale dans ce film. Notamment, c'est grâce à ces pensées joyeuses que Tootles se met à voler dans le ciel de Londres, faisant des pirouettes autour de Big Ben en hurlant : «I didn't lose my marbles after all! [...] Seize the day! » (Spielberg), et faisant ainsi écho à l'épicurisme de Peter Pan. De plus, ce sont ces pensées joyeuses qui font en sorte que Peter Banning accorde de nouveau une place dans son esprit au Peter Pan qui somnolait en lui, qu'il garde dès lors la fenêtre ouverte dans la chambre des enfants pour pouvoir regagner avec eux le Pays Imaginaire autant qu'il le voudra.

En dissociant ainsi corps et esprit, Spielberg illustre le fait que, bien que nous ne puissions éviter de vieillir, nous pouvons éviter de grandir. Alors que Barrie tolère cette éventualité chez Wendy, Disney s'abstient tout court de l'aborder. Spielberg, quant à lui, présente cette éventualité comme un idéal à atteindre, un idéal plus accessible que celui de Barrie ou de Disney, qui pour leur part privilégient le fantastique plutôt que le réel. Or, si ce mythe préfère l'enfance à l'âge adulte, c'est parce qu'un « central trope of "Peter Pan" in all its forms is that childhood represents a moment of privileged access to the imagination ${ }^{31}$ ", cette faculté qui nous permet de masquer les effets du temps sur notre personne. Par exemple, dans le film de Spielberg, le Peter Pan adulte ne peut se battre contre le capitaine Crochet que lorsqu'il a redécouvert le pouvoir de l'imaginaire du Peter Pan enfant. C'est donc dire que nous sommes sauvés par l'imaginaire par lequel le mythe 
nous encourage à nous laisser emporter. Toutefois, nous ne pouvons pas nous abandonner entièrement à notre imagination, puisque, contrairement aux personnages d'une histoire de fiction, nous sommes confrontés aux limites de la réalité, que nous pouvons seulement oublier par éclipses.

Ainsi, le mythe de Peter Pan, en plus de concéder au temps la puissance qui lui revient, nous indique des moyens de revendiquer une trêve à sa manifestation humaine. En effet, si l'usure de notre corps est inévitable, nous pouvons tout de même épargner notre esprit en sortant du temps historique, en quittant le monde duquel nous sommes prisonniers en lisant un roman, en regardant un film ou en jouant à un jeu, activités grâce auxquelles nous nous rendons compte que notre emprise sur le temps historique est insuffisante pour l'arrêter, certes, mais nous permet au moins de le ralentir.

\section{Conclusion}

41 Pour finir, si le mythe de Peter Pan bénéficie toujours d'une si grande popularité, c'est que les questions posées par le roman de Barrie, l'adaptation de Disney et la réécriture de Spielberg nous intéressent encore aujourd'hui. En effet, au même titre que le personnage de Peter Pan, le mythe de Peter Pan a réussi malgré les années à demeurer " exactly as fascinating as ever» (Barrie, p. 223) dans l'imaginaire collectif. Captant notre intérêt depuis plus d'un siècle, Peter Pan s'éloigne de notre monde et nous amène avec lui au Pays Imaginaire, un monde fantastique servant de refuge à un jeune garçon dont le souhait est "to always be a little boy and to have fun " (Barrie, p. 36), ainsi qu'à un terrible pirate qui est "afraid of time ticking away» (Spielberg). Là, les limites du possible éclatent et le temps gagne en élasticité.

Cependant, cette liberté du mythe est restreinte dans la réalité, faisant en sorte que nous voyons le temps comme une menace qu'il faut traiter avec prudence. Cela est d'autant plus évident lorsque Peter Pan avertit les garçons perdus qui souhaitent retourner à Londres pour y trouver des parents : « Once you've grown up, you can never come back... never! » (Disney) En d'autres mots, même l'enfant éternel sait que le temps et ses effets sont irréversibles. Toutefois, Spielberg défie cette idée en demandant dans la bandeannonce de Hook: «What if Peter Pan grew up? » (Spielberg) C'est alors qu'apparaît l'imaginaire en guise d'échappatoire. Il est donc clair que, même si chaque nouveau porte-parole du mythe doit trahir en quelque sorte la version fondatrice du mythe en modifiant certaines caractéristiques de l'histoire ou des personnages, ces modifications sont en fait la preuve qu'il y a toujours matière féconde à explorer et assurent de ce fait sa pérennité.

Or, «[s] usceptible de modifications [au fil du temps], adaptable, l'élément mythique est pourtant résistant dans le texte ${ }^{32} "$, car peu importe la version, Peter Pan nous met d'une part en garde contre la puissance du temps: "All children, except one, grow up." (Barrie, p.1) et célèbre d'autre part la ruse qu'il nous reste: interrompre le rythme effréné du temps historique grâce à l'imaginaire, qui suffit pour maintenir une part d'enfant en soi, même une fois adulte. Et bien que nous ne puissions pas nous envoler avec Peter Pan comme Wendy a pu le faire, nous pouvons accéder au même sentiment de légèreté en lisant leurs aventures ; des aventures qui nous font oublier le monde duquel nous faisons partie, jusqu'à perdre de vue la chaise dans laquelle nous sommes assis « en leur compagnie». Mais malgré ce sentiment, une mystérieuse vérité continue de nous inquiéter : «Ah me, how time flies!» (Barrie, p. 225) 


\section{BIBLIOGRAPHIE}

\section{Corpus d'analyse}

Barrie, J. M., 2006 [1911], Peter Pan, Oxford, Oxford University Press, 232 p.

Disney, Walt (dir.), 1953, Peter Pan, Disney Enterprises Inc.

Spielberg, Steven (dir.), 1991, Hook, TriStar Pictures.

\section{Articles cités}

Chevrel, Yves, 2005, « Réception et mythocritique » dans Danièle Chauvin, André Siganos et Philippe Walter (dir.), Questions de mythocritique. Dictionnaire, Paris, Éditions Imago, p. 283-294.

Friedman, Lester D, 2009, « Hooked on Pan : Barrie's Immortal Pirate in Fiction andFilm » dans Alison Kavey et Lester D. Friedman (éd.), Second Star to the Right: Peter Pan in the Popular Imagination, New Brunswick, N.J., Rutgers University Press, p. 188-218.

Geer, Jennifer, 2007, « J. M. Barrie Gets the Miramax Treatment : Finding (and Marketing) Neverland » dans Children's Literature Association Quarterly, vol. 32, no 3, p. 193-212. [en ligne, http://muse.jhu.edu/login?auth=0\&type=summary\&url=/journals/ childrens_literature_association_quarterly/v032/32.3geer.html]

Jack, R. D. S., 1994, « Peter Pan as Darwinian Creation Myth » dans Literature \& Theology: An International Journal of Theory, Criticism and Culture, vol. 8, $\mathrm{n}^{\circ}$ 2, p. 157-173.

Jack, R. D. S. 1990, « The Manuscript of Peter Pan » dans Children's Literature : Annual of The Modern Language Association Division on Children's Literature and The Children's Literature Association, vol. 18, p. 101-113. [En ligne : http://muse.jhu.edu/login?auth=0\&type=summary\&url=/journals/ childrens_literature/v018/18.jack.html] (Consulté le 23 juillet 2009.)

Molino, Jean, 1978, « Alexandre Dumas et le roman mythique », L'Arc, Aix-en Provence, $\mathrm{n}^{\circ} 71$, p. 56-69.

Ohmer, Susan, 2009, « Disney's Peter Pan : Gender, Fantasy, and Industrial Production » dans Alison Kavey et Lester D. Friedman (éd.), Second Star to the Right : Peter Pan in the Popular Imagination, New Brunswick, N.J., Rutgers University Press, p. 151-187.

Prévost, Maxime, 2008, « Compte rendu de Bayard (Pierre), L'Affaire du Chien des Baskerville » dans COnTEXTES, Notes de lecture. [En ligne : http://contextes.revues.org/index2783.html] (Consulté le 20 juillet 2009.)

Stoddard Holmes, Martha. 2009, «Peter Pan and the Possibilities of Child Literature » dans Alison Kavey et Lester D. Friedman (éd.), Second Star to the Right : Peter Pan in the Popular Imagination, New Brunswick, N.J., Rutgers University Press, p. 132-150.

\section{Ouvrages cités}

Blumenberg, Hans, 2005, La raison du mythe, Paris, Gallimard, 154 p.

Brunel, Pierre, 1992, Mythocritique. Théories et parcours, Paris, Presses universitaires de France, $294 \mathrm{p}$.

Brunel Pierre (dir.), 1988, Dictionnaire des mythes littéraires, Monaco, Éditions du Rocher, 1436 p. 
Campbell, Joseph, 1991, The Power of Myth, New York, Anchor Books, 233 p.

Eliade, Mircea, 1963, Aspects du mythe, Paris, Gallimard, 246 p.

Eliade, Mircea, 1957, Mythes, rêves et mystères, Paris, Gallimard, 310 p.

Jolles, André, 1972 [1930], Formes simples, trad. Antoine Marie Buguet, Paris, Éditions du Seuil, $212 \mathrm{p}$.

\section{NOTES}

1. Pierre Brunel, 1992. Mythocritique. Théories et parcours, Paris, Presses universitaires de France, p. 59.

2. Maxime Prévost, 2008, "Compte rendu de Bayard (Pierre), L'Affaire du Chien des Baskerville », COnTEXTES [En ligne], Notes de lecture, mis en ligne le 10 juillet 2008. (Consulté le 20 juillet 2009.)

3. Jean Molino, 1978, «Alexandre Dumas et le roman mythique », L'Arc (Aix-en-Provence), ${ }^{\circ} 71$, p. 57.

4. André Jolles, 1972 [1930], Formes simples, trad. Antoine Marie Buguet, Paris, Éditions du seuil, p. 81.

5. Hans Blumenberg, 2005, La raison du mythe, Paris, Gallimard, p. 69.

6. J. M. Barrie, 2006 [1911], Peter Pan, Oxford, Oxford University Press, 232 p.

7. Walt Disney (dir.), 1953, Peter Pan, Disney Enterprises Inc.

8. Steven Spielberg (dir.), 1991. Hook, TriStar Pictures.

9. Les titres des sections 1 à 3 renverront aux trois fonctions du mythe que Pierre Brunel élabore dans la préface à son Dictionnaire des mythes littéraires.

10. Pierre Brunel, 1992, Mythocritique. Théorie et parcours, Paris, Presses universitaires de France, p. 18. Notons que Barrie a contribué au mystère qui entoure l'intrigue de Peter Pan: "In the dedication to the play he contends, "I have no recollection of writing the play of Peter Pan" [...] The desire to conceal the power of his own art in order to glorify both the creative strength of motherhood [...] and the free imagination of youth is, for Barrie, not a whim but an important part of the myth. " (R. D. S. Jack, 1990, "The Manuscript of Peter Pan» dans Children's Literature: Annual of The Modern Language Association Division on Children's Literature and The Children's Literature Association [En ligne], vol. 18, p. 101.)

11. Mircea Eliade, 1963, Aspects du mythe, Paris, Gallimard, « Folio », p. 232.

12. R. D. S. Jack, 1994, «Peter Pan as Darwinian Creation Myth» dans Literature \& Theology: An International Journal of Theory, Criticism and Culture, vol. 8, no 2, p. 165.

13. D'après Eliade, "[1]'imitation des archétypes trahit un certain dégoût de sa propre histoire personnelle et la tendance obscure à transcender son moment historique local, provincial, et à recouvrer un "Grand Temps" quelconque [...]» (Mircea Eliade, 1957, Mythes, rêves et mystères, Paris, Gallimard, p. 33

14. Le vecteur mémoriel est l'œuvre responsable de la pérennité du mythe dans l'imaginaire collectif.

15. Susan Ohmer, 2009, «Disney's Peter Pan: Gender, Fantasy, and Industrial Production » dans Alison Kavey et Lester D. Friedman (éd.), Second Star to the Right: Peter Pan in the Popular Imagination, New Brunswick, N.J., Rutgers University Press, p. 170.

16. Joseph Campbell, 1991, The Power of Myth, New York, Anchor Books, p. 15.

17. Susan Ohmer, 2009, "Disney's Peter Pan: Gender, Fantasy, and Industrial Production » dans Alison Kavey et Lester D. Friedman (éd.), Second Star to the Right: Peter Pan in the Popular Imagination, New Brunswick, N.J., Rutgers University Press, p. 151-152. 
18. Martha Stoddard Holmes, 2009, «Peter Pan and the Possibilities of Child Literature » dans Alison Kavey et Lester D. Friedman (éd.), Second Star to the Right: Peter Pan in the Popular Imagination, New Brunswick, N.J., Rutgers University Press, p. 142.

19. Lester D. Friedman, 2009, "Hooked on Pan : Barrie's Immortal Pirate in Fiction and Film » dans Alison Kavey et Lester D. Friedman (éd.), Second Star to the Right: Peter Pan in the Popular Imagination, New Brunswick, N.J., Rutgers University Press, p. 196.

20. Yves Chevrel, 2005, «Réception et mythocritique » dans Danièle Chauvin, André Siganos et Philippe Walter (dir.), Questions de mythocritique. Dictionnaire, Paris, Éditions Imago, p. 284.

21. Pierre Brunel, 1988, Préface au Dictionnaire des mythes littéraires, Paris, Éditions du rocher, p. 7.

22. Joseph Campbell, 1991, The Power of Myth, New York, Anchor Books, p. 11.

23. Par " continuum du temps humain", nous entendons la gradation des traces que le temps laisse sur les hommes, c'est-à-dire les manifestations humaines du temps rendu visible avec l'âge. 24. Le capitaine Crochet doit son nom, son crochet, bref sa marque de commerce à cet épisode. C'est donc dire que ce personnage est prisonnier du temps qui l'effraie. Notons par exemple que, de la même manière qu'un pantin n'est pas maître de ce qui lui arrive, la moustache du capitaine Crochet se raidit comme une aiguille et vibre au rythme du temps lorsqu'il entend le tic tac du crocodile dans le dessin animé de Disney.

25. Lester D. Friedman, 2009, «Hooked on Pan : Barrie's Immortal Pirate in Fiction and Film » dans Alison Kavey et Lester D. Friedman (éd.), Second Star to the Right: Peter Pan in the Popular Imagination, New Brunswick, N.J., Rutgers University Press, p. 211.

26. Par grandir, nous entendons la mort de l'esprit, alors que vieillir renvoie davantage à la mort du corps.

27. Notons cependant que Spielberg a compris la valeur qu'accordait Barrie à l'éducation dans le passage de l'enfance à l'âge adulte : dans Hook, le capitaine Crochet tente d'élever les enfants du Peter Pan adulte en leur enseignant ses principes et maximes. Il fait ceci dans l'espoir de les enlever à son ennemi, coup qu'il réussit presque chez le plus vieux des deux enfants, mais qui échoue à la vue de son père enjoué.

28. R. D. S. Jack, 1990, «The Manuscript of Peter Pan » dans Children's Literature: Annual of The Modern Language Association Division on Children's Literature and The Children's Literature Association [En ligne], vol. 18, p. 105.

29. Jennifer Geer, 2007, «J. M. Barrie Gets the Miramax Treatment: Finding (and Marketing) Neverland » dans Children's Literature Association Quarterly, vol. 32, no 3, p. 198.

30. Notons que dans le nom Banning se cache le verbe to ban, illustrant que le Peter Pan adulte a banni le Peter Pan enfant au sein de lui.

31. Susan Ohmer, 2009, "Disney's Peter Pan : Gender, Fantasy, and Industrial Production » dans Alison Kavey et Lester D. Friedman (éd.), Second Star to the Right: Peter Pan in the Popular Imagination, New Brunswick, N.J., Rutgers University Press, p. 155.

32. Pierre Brunel, 1992, Mythocritique. Théories et parcours, Paris, Presses universitaires de France, p. 79.

\section{RÉSUMÉS}

Il arrive parfois dans la littérature qu'une histoire ou un personnage retienne particulièrement notre attention, ainsi que celle de toute autre personne, quel que soit son âge, son genre, son 
niveau d'éducation ou sa culture d'origine. Un tel degré de popularité, un tel pouvoir de séduction et une telle force d'attraction peuvent se traduire en un seul mot: mythe. Parmi ces célébrités inégalées se trouve Peter Pan. Que ce soit en lisant l'œuvre originale de James Barrie, l'adaptation cinématographique de Walt Disney ou la réinterprétation de Steven Spielberg, le lecteur/spectateur ne peut manquer de remarquer que Peter Pan raconte, révèle et explique à chaque fois une des plus grandes préoccupations de l'humanité : Qu'est-ce que le temps ? Et au coeur du mythe de Peter Pan, une question plus obsédante encore est posée: Pouvons-nous échapper aux prises du temps? Par la voie palliative de l'imaginaire, nous apprenons que les effets du temps sur l'homme sont inévitables, mais pas nécessairement dévorants.

In literature, it sometimes happens that a story or a character particularly catches our attention, as well as any other person's attention, no matter what their age, gender, level of education or native culture may be. Such a degree of popularity, a power of seduction and a compelling attraction can translate itself into a single word: myth. Among these unparalleled celebrities is Peter Pan. Whether it is while reading James Barrie's original novel, Walt Disney's cinematographic adaptation, or Steven Spielberg's reinterpretation, the reader/spectator can not miss the fact that Peter Pan each time relates, reveals and explains one of the greatest preoccupations of humanity: What is time? And at the heart of the myth of Peter Pan, an even more haunting question arises: Can we escape time's hold on us? Through the palliative ways of imagination, we learn that the effects of time on Man are inevitable, but not necessarily allconsuming.

\section{AUTEUR}

\section{AMÉLIE MAXWELL}

Doctorante, Université d'Ottawa 